\title{
The influence of molecular complexity on expanding flows of ideal and dense gases
}

\author{
J. Harinck, ${ }^{1}$ A. Guardone ${ }^{2}$ and P. Colonna ${ }^{1, a)}$ \\ ${ }^{1}$ Energy Technology Section, Process and Energy Department, Delft University of Technology, \\ Leeghwaterstraat 44, 2628 CA Delft, The Netherlands \\ ${ }^{2}$ Dipartimento di Ingegneria Aerospaziale, Politecnico di Milano, Via La Masa 34, 20156 Milano, Italy
}

(Received 11 August 2008; accepted 8 July 2009; published online 7 August 2009)

\begin{abstract}
This paper presents an investigation about the effect of the complexity of a fluid molecule on the fluid dynamic quantities sound speed, velocity, and Mach number in isentropic expansions. Ideal-gas and dense-gas expansions are analyzed, using the polytropic ideal gas and Van der Waals thermodynamic models to compute the properties of the fluid. In these equations, the number of active degrees of freedom of the molecule is made explicit and it is taken as a measure of molecular complexity. The obtained results are subsequently verified using highly accurate multiparameter equations of state. For isentropic expansions, the Mach number does not depend on the molecular weight of the fluid but only on its molecular complexity and pressure ratio. Remarkably enough, the Mach number can either increase or decrease with molecular complexity, depending on the considered pressure ratio. The exit speed of sound and flow velocity, however, are dependent on both molecular complexity and weight, as well as on the inlet total temperature. The exit flow velocity is found to be a monotonically increasing function of molecular complexity for all expansion ratios, whereas the speed of sound monotonically increases with molecular complexity only at high pressure ratios. The speed of sound is not monotone for pressure ratios around 3 , which leads to the Mach number being nonmonotone at pressure ratios around 10. It should be noted that the sound speed and flow velocity depend much more strongly on molecular weight than on molecular complexity, which in realistic expansions often obscures the influence of the latter. Quantitative differences are observed between ideal and dense-gas expansions, which are dependent on the reduced inlet conditions. The present study concludes with the numerical simulation of two-dimensional expansions in a turbine nozzle to document the occurrence of real-gas effects and their dependence on molecular complexity in realistic applications. (c) 2009 American Institute of Physics. [DOI: 10.1063/1.3194308]
\end{abstract}

\section{INTRODUCTION}

Gas expansions are common processes in numerous industrial applications. Examples can be found in the power, oil and gas, chemical, process, and refrigeration industries. Fluids are expanded in order to generate mechanical power (e.g., in turbines, rockets, mixers, wind tunnels for aerodynamic testing, and for material removal). Fluid expansions are also used in order to reduce the pressure and/or temperature (e.g., for refrigeration purposes or to separate heavier fluid fractions from lighter ones by condensation). In general terms, the process conditions, namely, the initial thermodynamic state and expansion ratio, together with the fluid characteristics determine the dynamics of an expansion.

The choice of the working fluid is driven by different requirements arising from the specific application. A great variety of substances, with different thermophysical properties, are used in industry, ranging from the simple monatomic fluids to the heavy fluids formed by complex molecules. An example is the Rankine power cycle, in which, for largescale applications, the common working medium is steam, but for small-scale and low-temperature applications, organic

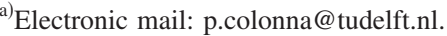

fluids such as hydrocarbons, perfluorocarbons, or siloxanes are more suitable. ${ }^{1-3}$ Gases made of heavy molecules can be used in supersonic wind tunnels instead of air to achieve higher Reynolds numbers which can be varied almost independently from the Mach number. ${ }^{4-6}$ Complex fluids with low boiling point are used also as working fluids in refrigeration and air conditioning equipment, and in heat pumps. For certain energy applications, even mixtures of fluids could be used to further tailor the fluid thermophysical properties to the requirements and to optimize the thermodynamic cycle-see, e.g., Ref. 3.

The process conditions can vary from expansions entirely embedded in the ideal-gas thermodynamic region, i.e., the region where volumetric properties can be calculated from the ideal-gas equation of state $P v=R T$, where $P$ denotes pressure, $v$ specific volume, $R$ the specific gas constant, and $T$ temperature, to those occurring close to the liquidvapor saturation line and to the critical point, namely, within the so-called dense-gas thermodynamic region. The latter is, for example, the case for supercritical $\mathrm{CO}_{2}$ nozzle flows, which are increasingly adopted in the pharmaceutical industry for the nucleation of chemicals. ${ }^{7,8}$ Exemplary ideal- and dense-gas expansion processes are represented by the isentropes depicted in the reduced pressure-specific-volume dia- 


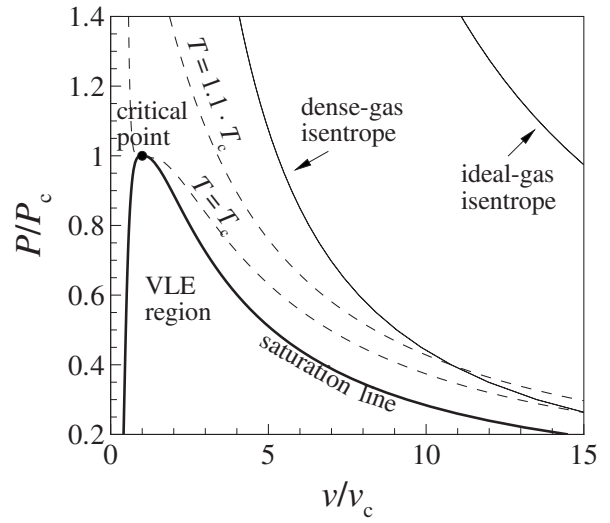

FIG. 1. The reduced pressure-specific-volume $v / v_{c}-P / P_{c}$ diagram, with $v_{c}$ and $P_{c}$ as the critical specific volume and pressure, respectively, of water based on the Wagner-Pruß thermodynamic model (Ref. 9) showing an isentrope in the ideal-gas region and one in the dense-gas region. The vaporliquid equilibrium region is bounded by the liquid-vapor saturation curve. The critical isotherm $T=T_{c}$ and the isotherm $T=1.1 T_{c}$ are also indicated.

gram of an arbitrary fluid in Fig. 1. In the dense-gas region, the actual thermodynamic behavior of gases can deviate significantly from that predicted by the ideal polytropic (constant isobaric and isochoric specific heat) gas law. These deviations in turn lead to deviations in fluid dynamics quantities, which are known as real-gas effects. Hence, more complex thermodynamic models must be used to complement the fluid dynamic analysis of a dense-gas process. ${ }^{10-13}$

Recent gas dynamic studies have focused on the influence of fluid molecular characteristics on the real-gas behavior of a fluid flow. Investigations in Ref. 14 concluded, based on a sensitivity analysis using various fluids, that real-gas behavior is strongly influenced by the isochoric specific heat per unit mole and by the acentric factor, which are both qualitative measures of molecular complexity. Here molecular complexity indicates the effect of both molecular configuration and molecular interactions. In Ref. 15, the authors derived, by means of the physically based Van der Waals equation of state (EoSs), the relation between the molecular complexity - defined as the capacity of the fluid molecules to store energy into the internal vibrational modes - and the derivative of the sound speed along isentropes, whose value influences real-gas behavior.

The aim of this work is to relate the fluid dynamic quantities sound speed, velocity, and Mach number along isentropic expansions to the fluid molecular characteristics and to the molecular complexity in particular. The influence of molecular complexity is analyzed for fluids being expanded in the ideal-gas thermodynamic region and in the (mildly nonideal) dense-gas region. The study starts with a nonstandard interpretation of well-known results of the polytropic idealgas law. The discussion then moves to the physically based but still relatively simple Van der Waals EoS, which allows for a treatment based on physical grounds. These results are then verified using highly accurate empirical EoSs. The study concludes with the numerical simulation of twodimensional (2D) expansions in a turbine nozzle, to document the occurrence of real-gas effects and their dependence on molecular complexity in realistic applications.
This paper is structured as follows. Section II introduces the fundamental derivative of gas dynamics and shows how it relates to molecular complexity quantified by the number of active degrees of freedom of a fluid molecule. In Sec. III, the effect of molecular complexity on the Mach number, flow velocity, and sound speed is analyzed for isentropic expansions in the ideal-gas and dense-gas thermodynamic regions. In Sec. IV the influence of molecular complexity on expansions is furthermore exemplified by presenting the fluid dynamic simulations of expansions of three fluids of practical interest, namely, steam, carbon dioxide and propane, which are conveniently different in molecular complexity and weight. Concluding remarks complete this work.

\section{MOLECULAR COMPLEXITY AND THE FUNDAMENTAL DERIVATIVE OF GASDYNAMICS}

In 1971 Thompson, ${ }^{16}$ following the work of Bethe, ${ }^{17}$ formally introduced the so-called fundamental derivative of gas dynamics $\Gamma$,

$$
\Gamma \equiv 1-\frac{v}{c}\left(\frac{\partial c}{\partial v}\right)_{s},
$$

where $v$ is the specific volume, $c$ is the sound speed, and $s$ is the specific entropy. The value of $\Gamma$ can be used to classify gas dynamic behavior. For an ideal polytropic gas, $\Gamma$ is constant and reduces to $\Gamma=(\gamma+1) / 2$, where $\gamma$ denotes the (constant) ratio of the isobaric to the isochoric specific heat. Therefore, the speed of sound derivative with respect to the volume in isentropic expansions reads $(\partial c / \partial v)_{s}$ $=(c / v)(1-\gamma) / 2<0$ and $\Gamma>1$ since $\gamma>1$ for thermodynamic stability.

Modern thermodynamic models indicate that the minimum value of $\Gamma$ in the single-phase vapor region for a given fluid decreases as the molecular complexity of the fluid increases. ${ }^{14}$ The above can be appreciated already in the case of an ideal polytropic gas, for which molecular complexity can be conveniently defined as the number $N$ of active degrees of freedom of the molecule. ${ }^{15}$ In the temperature range of interest here, these are the translational, rotational, and vibrational degrees of freedom. Both the translational and the rotational modes are assumed to be fully excited, whereas the activation of the vibrational ones occurs only at temperatures higher than the vibrational temperature associated with each mode, hence, $N=N(T)$. According to the energy equipartition principle, ${ }^{18}$ at a given temperature, each fully active degree of freedom contributes $R / 2$, where $R$ is the specific gas constant, to the overall value of the ideal-gas isochoric specific heat, $C_{v}^{0}$, so that

$$
C_{v}^{0}(T)=\frac{\bar{R}}{2} \frac{N(T)}{M},
$$

where $\bar{R}$ is the universal gas constant equal to $\bar{R}=8.314 \mathrm{~J} \mathrm{~mol}^{-1} \mathrm{~K}^{-1}$ and $M$ is the molecular weight. In this work, the number of active degrees of freedom of a fluid is evaluated at its critical temperature, denoted by $T_{c}$, i.e., $N=2 M C_{v}^{0}\left(T_{c}\right) / \bar{R}$. Therefore, $\gamma=1+2 / N$ and the (complete) polytropic ideal-gas (PIG) model reads 
PIG: $\quad P(T, v)=\frac{1}{M} \frac{\bar{R} T}{v}, \quad e(T)=\frac{N}{M} \frac{\bar{R} T}{2}+e_{0}$,

where $e$ is the specific internal energy per unit mass and subscript 0 denotes its value at an arbitrary reference state. The fundamental derivative for an ideal polytropic gas is then

$$
\text { PIG: } \quad \Gamma \equiv 1+\frac{1}{N},
$$

and its value, which is constant for a given fluid, decreases with increasing molecular complexity.

In the dense-gas region $\Gamma$ is no longer a constant and its value depends on the thermodynamic state as well as on the fluid properties. For example, in the polytropic Van der Waals ${ }^{19}$ (PVdW) model,

$$
\begin{aligned}
\mathrm{PVdW}: \quad P(T, v) & =\frac{1}{M} \frac{\bar{R} T}{v-b}-\frac{a}{v^{2}}, \\
e(T, v) & =\frac{N}{M} \frac{\bar{R} T}{2}-\frac{a}{v}+e_{0},
\end{aligned}
$$

the effects of long- and short-range intermolecular forces are included via the two fluid-dependent parameters $a=3 P_{c} v_{c}^{2}$ and $b=v_{c} / 3$, respectively, where the subscript $\mathrm{c}$ indicates critical point quantities. Note that the Van der Waals model can be written in a so-called reduced form,

$$
\begin{aligned}
\mathrm{PVdW}: \quad \tilde{P}(\widetilde{T}, \widetilde{v}) & =\frac{8 \widetilde{T}}{3 \widetilde{v}-1}-\frac{3}{\widetilde{v}^{2}}, \\
\widetilde{e}(\widetilde{T}, \widetilde{v}) & =\frac{4}{3} N \widetilde{T}-\frac{3}{\widetilde{v}}+\widetilde{e}_{0},
\end{aligned}
$$

where $\tilde{P}=P / P_{c}, \widetilde{T}=T / T_{c}, \widetilde{v}=v / v_{c}$, and $\tilde{e}=e /\left(P_{c} v_{c}\right)$ in which the parameters $M, a$, and $b$ are no longer present and the only substance-specific quantity is the molecular complexity $N$. Therefore, the fundamental derivative of a PVdW gas is a decreasing function of the molecular complexity $N$ and its value depends also on the reduced thermodynamic state, e.g., $(\widetilde{T}, \widetilde{v})$, as follows: ${ }^{15}$

$$
\mathrm{PVdW}: \quad \Gamma(\widetilde{T}, \widetilde{v})=\frac{\left(1+\frac{1}{N}\right)\left(1+\frac{2}{N}\right) \frac{3 \tilde{T} \tilde{v}}{(3 \tilde{v}-1)^{3}}-\frac{1}{\widetilde{v}^{3}}}{\left(1+\frac{2}{N}\right) \frac{\tilde{T}}{(3 \widetilde{v}-1)^{2}}-\frac{2}{3} \frac{1}{\widetilde{v}^{3}}} .
$$

More complex models, such as the multiparameter EoSs (MPEoSs) include a large number of fluid-dependent parameters, which in turn influence the evaluation of the fundamental derivative $\Gamma$.

Multiparameter equations of state (MPEoSs) can be classified as technical or reference equations of state. ${ }^{20,21}$ Both reference and technical equations of state are able to describe all the best experimental thermodynamic property data available for a certain fluid within their experimental uncertainty. Reference equations of state have been developed only for few substances, simple gases, and water, for which a large number of accurately evaluated experimental data of all kinds of thermodynamic properties are available (hundreds or thousands of data whose high accuracy has been determined). Their development requires therefore a large amount of work because the functional form of the equation, specialized to the fluid, is also optimized. Technical equations of state fulfill to a lower set of requirements and are therefore less accurate, but they are able to satisfy the demand of modern technical applications. Their functional form is simpler and it is not fluid specific, but rather applies to a class of substances. Still the relatively large number of parameters allow for the correct fitting of a wide variety of experimental points (liquid and vapor pressure-volume-temperature points, saturation pressure and density, enthalpies of vaporization, speed of sound, etc.). The main limitation of MPEoSs is that their development requires the availability of a set of carefully evaluated experimental data of many different thermodynamic properties. For this reason, the number of fluids for which reference or technical equations of state is available is still quite limited (six references and 30 technical equations of state). Another drawback of MPEoSs is that their empirical nature prevents from assigning a physical significance to parameters, as opposed, for instance, to the Van der Waals family of cubic equations of state.

An example is the Wagner-Pruß MPEoS for water, ${ }^{9}$ for which the uncertainty in the vapor phase is less than $0.1 \%$ and $0.2 \%$ for all volumetric and caloric properties, respectively. By comparison, the MPEoSs for carbon monoxide, toluene, and R245fa are technical and their accuracy is between $0.2 \%$ and $1 \%$ for volumetric properties and $2 \%$ for caloric properties. ${ }^{22}$ In this work, the MPEoSs implemented in the REFPROP code ${ }^{23}$ by NIST have been used.

It is worthwhile pointing out that if evaluated from a highly accurate thermodynamic model such as a MPEoS, the fundamental derivative of gas dynamics $\Gamma$ is a more general parameter than the number of active degrees of freedom $N$, if used as a measure of molecular complexity. Indeed, $\Gamma$ quantifies the thermodynamic effect of all possible mechanism for storing energy, thereby implicitly also taking into account the contribution from intermolecular attractive and repulsive forces to the isochoric heat capacity, such as the aforementioned Coulomb, dipole-dipole, three and many body potentials. In this respect, the following classification was proposed in Ref. 15. Fluids with $\Gamma>1$ everywhere $\left[(\partial c / \partial v)_{s}\right.$ $<0]$ in the vapor thermodynamic region are classified as low molecular complexity (LMC) fluids. ${ }^{15,16}$ For LMC fluids, which are made of simple molecules, real-gas effects are limited to quantitative deviations with respect to ideal-gas fluid dynamic behavior, ${ }^{24}$ an exception being the temperature increase due to the Joule-Thompson isenthalpic expansion above a fluid's inversion temperature. Similarly, fluids with $0<\Gamma<1$ in a finite thermodynamic region are termed high molecular complexity (HMC) fluids since $(\partial c / \partial v)_{s}>0$. For HMC fluids also qualitative differences in fluid dynamic behavior can be observed in the dense-gas region, close to saturation. ${ }^{25-27}$ Most notable is the increase in the speed of sound upon isentropic expansion, possibly leading to a local decrease in the Mach number, ${ }^{11,27-29}$ which instead increases 


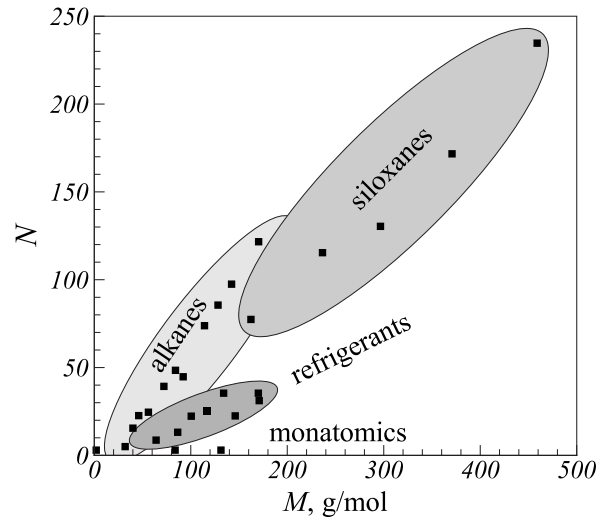

FIG. 2. Molecular complexity (in terms of the number of active degrees of freedom) vs molecular weight for selected fluids listed in Table I.

monotonically for an isentropic ideal-gas expansion. Reference 25 reports examples of expansions of a group of alkanes in the dense-gas region. Finally, so-called Bethe-Zel'dovich-Thompson ${ }^{17,30,31}$ (BZT) fluids exhibit a (smaller) finite thermodynamic region where $\Gamma<0 .{ }^{32} \mathrm{BZT}$ fluids are made of complex molecules such as perfluorocarbon PP10 $\left(\mathrm{C}_{13} \mathrm{~F}_{22}\right)$ or siloxane $\mathrm{D}_{6}\left(\mathrm{C}_{12} \mathrm{H}_{36} \mathrm{Si}_{6} \mathrm{O}_{6}\right)$, nonclassical gas dynamic phenomena including rarefaction shock waves $^{33}$ may possibly occur, although no experimental evidence of these "exotic" wave fields is available yet. ${ }^{34}$

In Ref. 14, the dependence of $\Gamma$ on selected fluid parameters including the acentric factor, the critical compressibility, and the assumption of polytropicity has been assessed using several simple thermodynamic models. It was concluded that within the same fluid family or class, the minimum value of $\Gamma$ depends on the ratio of the isochoric heat capacity to the gas constant $R$. In the nomenclature used here, this translates into the fact that the molecular complexity of a fluid family depends only on the active degrees of freedom $N$. In Ref. 31, the observation that, for a given reduced thermodynamic state, $\Gamma$ decreases with increasing $N$ [cf. Eq. (6)] is demonstrated to hold also for a general EoS. In the following, $N$ is the only parameter used to indicate molecular complexity.

For a given fluid family or class, molecular weight and molecular complexity are related because their molecular structures do not vary. Within a single fluid family, an increase in the number of active degrees of freedom is proportional to an increase in molecular weight, at least for the heavier fluids belonging to the family. The value of the complexity-to-weight ratio $N / M$ depends on the molecular structure of the fluid family, i.e., on the weight and configuration of the individual atoms in the molecule, and is therefore different for each class of fluids, as shown for a selection of fluids in Fig. 2. The figure sets into evidence that molecules of high-molecular-weight fluids often also have a high number of degrees of freedom (HMC). Note that this is true to some extent also for fluids belonging to different families. The correlation of molecular complexity and weight makes it difficult to attribute fluid dynamic properties to these properties separately, and hence these terms are often used interchangeably in literature, even though this practice is questionable if different fluid families are considered. ${ }^{25,28}$ In Sec. III the separate influence of the values of $N$ and $M$ on isentropic expansions is investigated.

\section{INFLUENCE OF MOLECULAR COMPLEXITY AND WEIGHT ON ISENTROPIC EXPANSIONS}

In this section, the influence of molecular complexity and weight on the speed of sound $c$, the flow velocity $u$, and the Mach number $\mathrm{Ma}=u / c$ along isentropic expansions is investigated. The flow is assumed to undergo a steady isentropic expansion from an infinitely large reservoir, where the fluid is at rest and at so-called reservoir or total conditions, into an ambient where the pressure is uniform. For the expansion to be isentropic, the hypothesis is that shock waves are absent and the effects of viscosity and thermal conductivity can be neglected, which is often the case at high Reynolds number, if no separation occurs. Isentropic conditions are therefore often assumed during the preliminary design of any expander and also in the following. Finally, the flow is assumed to be quasi-one-dimensional, allowing for a scalar velocity $u$ to represent the average axial velocity.

First, the procedure to compute $c, u$, and $\mathrm{Ma}$ is presented for a general EoS and it is then specialized to the case of ideal polytropic gases in Sec. III A and of dense gases in Sec. III B. The properties of dense gases are modeled using both the Van der Waals and very accurate multiparameter EoSs.

Given an arbitrary pressure-explicit equation of state $P=P(T, v)$, the sound speed $c$ is given by

$$
c^{2} \equiv\left(\frac{\partial P}{\partial \rho}\right)_{s}=-v^{2}\left[\left(\frac{\partial P}{\partial v}\right)_{T}-\frac{T}{C_{v}}\left(\frac{\partial P}{\partial T}\right)_{v}^{2}\right],
$$

where the density $\rho=1 / v$. The isochoric specific heat capacity is computed as the sum of an ideal-gas contribution $C_{v}^{0}=C_{v}^{0}(T)$ and a real-gas residual that is only dependent on the pressure EoS, i.e., ${ }^{35}$

$$
C_{v}(T, v)=C_{v}^{0}(T)+T \int_{\infty}^{v}\left(\frac{\partial^{2} P}{\partial T^{2}}\right)_{v} d v .
$$

Note that even if $C_{v}^{0}$ is independent from $T$ under the polytropic assumption, the real gas $C_{v}$ is not constant and depends on both the temperature and the specific volume.

The fluid velocity can be computed from the energy conservation for an isentropic transformation, which implies that the total or stagnation enthalpy $h^{t}$ is constant. Therefore, if $h_{1}^{t}$ is the total enthalpy at the inlet of the one-dimensional channel, the so-called inlet or reservoir total enthalpy, then from the definition of $h^{t}, h^{t}=h+u^{2} / 2$, with $h=e+P v$ the specific enthalpy, one immediately obtains $u^{2}=2\left(h_{1}^{t}-h\right)$. In particular, if one considers an isentropic expansion to an outlet pressure $P_{2}$, with the outlet state being denoted by subscript 2 , then the flow velocity at the end of the expansion reads

$$
u_{2}^{2}=2\left(h_{1}^{t}-h_{2}\right),
$$

where the exit enthalpy $h_{2}$ is computed as $h_{2}=h\left(s_{1}, P_{2}\right)$ since $s=s_{1}=$ const under the isentropic assumption. The exit or outlet speed of sound and Mach number are computed as function of $s_{1}$ and $P_{2}$ as well. In Secs. III A and III B results for ideal and dense-gas flows are given. 

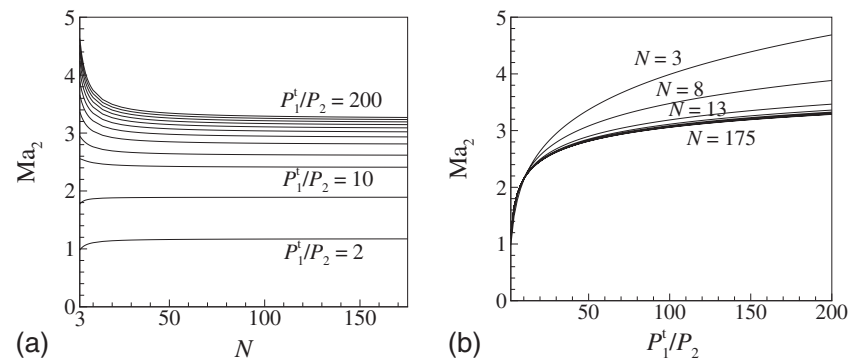

FIG. 3. Influence of (a) molecular complexity and (b) pressure ratio on the exit Mach number $\mathrm{Ma}_{2}$ for fluids expanded in the ideal-gas region, as given by Eq. (10).

\section{A. Polytropic ideal gas}

In case of expansions occurring in the dilute-gas thermodynamic region, the PIG law defined in Eq. (3) can be used to quantify the influence of the molecular complexity and weight on the flow field. By substituting $\gamma=1+2 / N$ and $R=\bar{R} / M$ into the well-known relations for Ma, $c$, and $u$ for steady isentropic flows of polytropic ideal gases-see, e.g., Ref. 32-the dependence of the flow quantities on the molecular complexity $N$ and weight $M$ can be derived immediately. For the Mach number Ma one has

$$
\mathrm{Ma}_{2, \mathrm{PIG}}^{2}=N\left[\left(\frac{P_{1}^{t}}{P_{2}}\right)^{2 /(N+2)}-1\right],
$$

an expression that places into evidence the dependence of the exit Mach number on molecular complexity and shows as a rather unpredictable result that $\mathrm{Ma}_{2}$ does not depend directly on the molecular weight of the fluid. It is remarkable that such a well-known relation, commonly written using $\gamma$, has still some information to unveil if the simple substitution $\gamma=1+2 / N$ is made.

The exit Mach number depends of course also on the pressure or expansion ratio $P_{1}^{t} / P_{2}$, where $P_{1}^{t}$ is the inlet total pressure. Figures 3(a) and 3(b) report the exit Mach number for different values of molecular complexity and expansion ratio. The dependence on $P_{1}^{t} / P_{2}$ is relevant for all considered pressure ratios; as well known, the larger the expansion ratio, the higher the exit Mach number. The effect of molecular complexity is large from $N=3$ up to $N \approx 30$. Remarkable enough, the Mach number can either increase or decrease with molecular complexity, depending on the considered pressure ratio. This is evident in Fig. 3(a), where the $P_{1}^{t} / P_{2} \approx 10$ curve separates the two trends. More precisely, for low pressure ratios, the Mach number is a monotonically increasing function of $N$. For higher pressure ratios, the Mach number first sharply increases to a maximum between approximately $0<N<5$ and then decreases for fluids of increasing molecular complexity. The occurrence of a maximum is the result of the counteracting influences of $N$ in Eq. (10). Above the minimum number of degrees of freedom of a molecule, i.e., $N=3$ corresponding to a monatomic gas, the Mach number is a decreasing function of $N$ at these high pressure ratios. The locus of the Mach number extrema is given in Fig. 4. Figure 4 can be used to determine the effect of a change in the fluid properties $(N)$ or pressure ratio on the

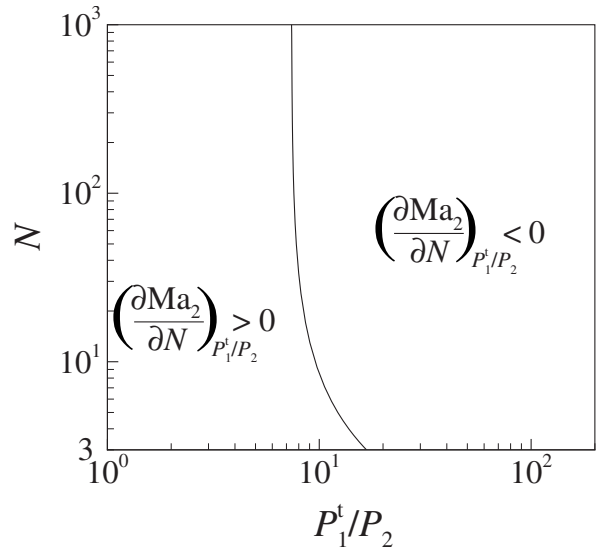

FIG. 4. Locus of exit Mach number maxima at fixed pressure ratios.

exit Mach number. For example, if the operating conditions are in the upper right portion of the diagram, then $\left(\partial \mathrm{Ma}_{2} / \partial N\right)_{P_{1}^{t} / P_{2}}<0$ and employing a more complex fluid would lead to a decrease in the exit Mach number, and vice versa for the left part.

Considering now the sound speed, from Eq. (7), the ideal polytropic gas sound speed reduces to

$$
c_{2, \mathrm{PIG}}^{2}=\gamma R T_{2}=\left(1+\frac{2}{N}\right) \frac{\bar{R} T_{1}^{t}}{M}\left(\frac{P_{1}^{t}}{P_{2}}\right)^{-2 /(N+2)},
$$

where $T_{1}^{t}$ is the total (stagnation) temperature at inlet. In the above expression, the term $(1+2 / N) \bar{R}$ is the molar specific heat at constant pressure which decreases with increasing molecular complexity. The term $1 / M$ decreases with the molecular weight. Increasing the inlet total temperature $T_{1}^{t}$ results in an increase in the exit speed of sound. Finally, the term $\left(P_{1}^{t} / P_{2}\right)^{-2 /(N+2)}=T_{2} / T_{1}^{t}$ is a positive increasing function of $N$ since $P_{1}^{t} / P_{2}>1$. This contribution is due to the temperature ratio resulting from a given expansion ratio, which is higher for complex fluids, i.e., the outlet temperature is closer to the inlet value, a result that is confirmed also by more complex thermodynamic models.

The flow velocity (9) reads instead

$$
u_{2, \mathrm{PIG}}^{2}=2\left(h_{1}^{t}-h_{2}\right)=(N+2) \frac{\bar{R} T_{1}^{t}}{M}\left[1-\left(\frac{P_{1}^{t}}{P_{2}}\right)^{-2 /(N+2)}\right] \text {, }
$$

where the PIG relations $h-h_{\text {ref }}=C_{P}^{0}\left(T-T_{\text {ref }}\right)$, with $h_{\text {ref }}=0 \mathrm{~J} / \mathrm{kg}$ at $T_{\text {ref }}=0 \mathrm{~K}$ have been applied. Therefore, two contributions from molecular complexity can be singled out. The first term, $N+2$, is in fact $2 C_{P}^{0} \bar{R} / M$, and it is linear with $N$. The term $\left(1-P_{1}^{t} / P_{2}\right)^{-2 /(N+2)}$ is the relative temperature difference between the total inlet temperature and the outlet temperature, which is a mildly decreasing function of $N$.

Relations (11) and (12) show that the exit speed of sound and flow velocity are dependent on both molecular complexity $N$ and weight $M$, as well as on the inlet total temperature $T_{1}^{t}$. To remove the dependence on $M$ and $T_{1}^{t}$, the exit speed of sound and flow velocity are made dimensionless by $\sqrt{\left(\bar{R} T_{1}^{t} / M\right)}$ and plotted in Fig. 5 for different expansion ra- 

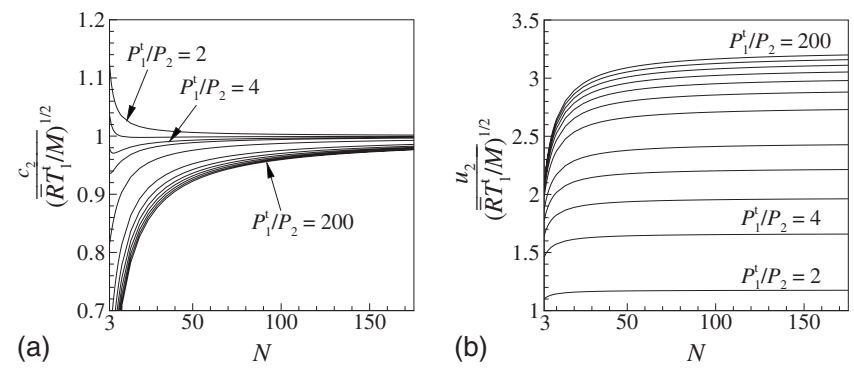

FIG. 5. Dimensionless outlet sound speed and velocity for fluids with different molecular complexities expanded in the ideal-gas region at various expansion ratios. The reference velocity used in the adimensionalization is $\sqrt{R} T_{1}^{t} / M$ to remove the dependence on both the reservoir state and the molecular weight.

tios and for fluids with different molecular complexity. The dimensionless exit flow velocity is found to be a monotonically increasing function of $N$ regardless of the expansion ratio, whereas the speed of sound increases monotonically with $N$ for $P_{1}^{t} / P_{2}$ larger than about 3 . The nonmonotone behavior of the speed of sound explains the similar trend of the Mach number shown in Fig. 3(a).

The influence of the molecular weight $M$ can be appreciated from Fig. 6, where the dimensional sound speed and velocity at the outlet are plotted for $T_{1}^{t}=322.5 \mathrm{~K}$ and for different values of $N$ and $M$. Three different pressure ratios are considered. Due to the presence of the term $1 / M$ in expressions (11) and (12), both $c_{2}$ and $u_{2}$ are monotonic decreasing functions of $M$. Note again the reversed behavior with respect to $N$ of the sound speed for pressure ratios larger than about 3 . For the physically relevant interval of $3<N<200$ - which for the selected fluids corresponds to approximately $0<M<500 \mathrm{~g} / \mathrm{mol}$, see Fig. 2 and Table I-the sound speed depends much more strongly on molecular weight than on molecular complexity, as shown in Fig. 6(a).

\section{B. Dense-gas region}

To analyze the effect of the molecular complexity in the dense-gas thermodynamic region, where the PIG assumption is no longer valid, two types of thermodynamic models are employed here, the PVdW (Ref. 19) EoS model (4) and a MPEoSs.
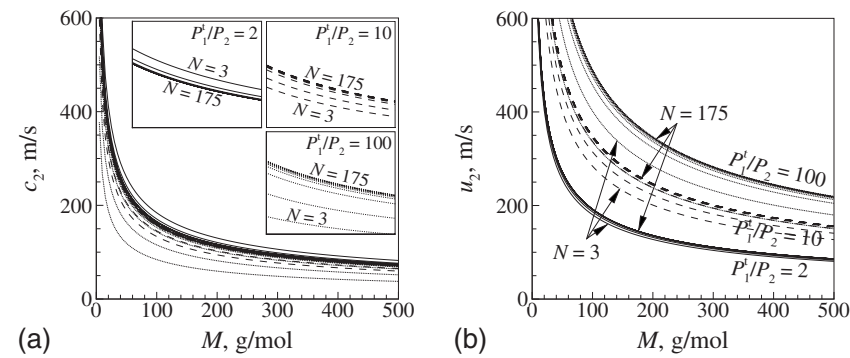

FIG. 6. Outlet sound speed and flow velocity as functions of molecular weight for fluids of various molecular complexity and for three different expansion ratios. The total temperature at the inlet of the one-dimensional channel is $322.5 \mathrm{~K}$.
The sound speed relation at the outlet for a PVdW fluid is obtained by substituting the model definition (4) into relation (7) which gives

$$
c_{2, \mathrm{PVdW}}^{2}=\frac{\bar{R} T_{c}}{M}\left[\left(1+\frac{2}{N}\right) \widetilde{T}_{1}^{t}\left(\frac{3 \widetilde{v}_{1}^{t}-1}{3 \widetilde{v}_{2}-1}\right)^{2 / N}\left(\frac{3 \widetilde{v}_{2}}{3 \widetilde{v}_{2}-1}\right)^{2}-\frac{6}{\widetilde{v}_{2}}\right]
$$

where in the second expression, the isentropic assumption, $s\left(T_{1}^{t}, v_{1}^{t}\right)=s\left(T_{2}, v_{2}\right)$, with

$$
s(T, v)=s_{0}+\frac{N}{M} \frac{\bar{R}}{2} \ln \frac{T}{T_{0}}+\frac{\bar{R}}{M} \ln \frac{v-b}{v_{0}-b},
$$

here $s_{0}, T_{0}$, and $v_{0}$ are evaluated at a suitably defined reference state, has been used to compute the outlet temperature in terms of the stagnation inlet temperature and specific volume. In the last expression of Eq. (13), the temperature and the specific volume are made dimensionless (reduced) with their corresponding critical point values. The total specific volume at inlet $v_{1}^{t}$ is computed using the Van der Waals pressure EoS, namely, by solving for $v_{1}^{t}$ the equation $P\left(T_{1}^{t}, v_{1}^{t}\right)$ $-P_{1}^{t}=0$. The outlet specific volume $v_{2}$ is instead computed using the isentropic assumption $s\left(P_{1}^{t}, v_{1}^{t}\right)=s\left(P_{2}, v_{2}\right)$, where

$$
s(P, v)=s_{0}+\frac{N}{2} \frac{\bar{R}}{M} \ln \left[\frac{M\left(P+\frac{a}{v^{2}}\right)(v-b)^{1+(2 / N)}}{\bar{R} T_{0}\left(v_{0}-b\right)^{2 / N}}\right] .
$$

Similarly, the exit velocity is computed from Eqs. (9) and (4) as

$$
\begin{aligned}
u_{2, \mathrm{PVdW}}^{2}= & 2\left[h_{1}^{t}-h_{2}\right]=\frac{\bar{R} T_{c}}{M}\left[\widetilde { T } _ { 1 } ^ { t } \left\{\frac{N}{2}\left[1-\left(\frac{3 \widetilde{v}_{1}^{t}-1}{3 \widetilde{v}_{2}-1}\right)^{2 / N}\right]\right.\right. \\
& \left.\left.+\frac{3 \widetilde{v}_{1}^{t}}{3 \widetilde{v}_{1}^{t}-1}\left(\frac{3 \widetilde{v}_{1}^{t}-1}{3 \widetilde{v}_{2}-1}\right)^{2 / N} \frac{3 \widetilde{v}_{2}}{3 \widetilde{v}_{2}-1}\right\}-6 \frac{\widetilde{v}_{1}^{t}-\widetilde{v}_{2}}{\widetilde{v}_{1}^{t} \widetilde{v}_{2}}\right],
\end{aligned}
$$

where the expression of the enthalpy for a PVdW fluid,

$$
h(T, v)=R T\left[\frac{N}{2}+\frac{v}{v-b}\right]-2 \frac{a}{v}+e_{0},
$$

has been used to compute the difference between the inlet total and the outlet enthalpy.

In a given reduced state, the exit sound speed $c_{2}(13)$ and flow velocity $u_{2}$ (14) depend on the molecular complexity $N$, on the molecular weight $M$, as well as on the critical temperature of the fluid $T_{c}$. The dependence on $M$ is the same as in the ideal-gas case and both the speed of sound and the flow velocity decrease with $M$. Instead, $c_{2}$ and $u_{2}$ increase with $T_{c}$, which is higher for complex molecules. Figure 7 reports the critical temperature of the fluids listed in Table I as a function of molecular complexity. From relations (13) and (14), the exit Mach number is found to be independent of either the molecular weight $M$ or the critical temperature $T_{c}$, as in the ideal-gas case.

In the following, to eliminate the dependence on $M$ and $T_{c}$, the speed of sound and the flow velocity are made dimensionless by means of the reference velocity $\sqrt{\bar{R} T_{c} / M}$. How- 
TABLE I. Fluids used in the dense-gas study (Figs. 8-10). The number of active degrees of freedom of these fluids is plotted against the molecular weight in Fig. 2 and their critical temperature vs their number of active degrees of freedom in Fig. 7. Information about the source of experimental data and accuracy of the MPEoS can be found in Ref. 23.

\begin{tabular}{|c|c|c|c|c|c|}
\hline Name & Chemical formula & $\begin{array}{c}M \\
(\mathrm{~g} / \mathrm{mol})\end{array}$ & $N(-)$ & $\begin{array}{c}P_{c} \\
\text { (bar) }\end{array}$ & $\begin{array}{c}T_{c} \\
\left({ }^{\circ} \mathrm{C}\right)\end{array}$ \\
\hline Hydrogen & $\mathrm{H}_{2}$ & 2.02 & 3.00 & 13.0 & -240 \\
\hline Parahydrogen & $p-\mathrm{H}_{2}$ & 2.02 & 3.00 & 12.9 & -240 \\
\hline Helium & $\mathrm{He}$ & 4.00 & 3.00 & 2.27 & -268 \\
\hline Deuterium & $\mathrm{D}$ & 4.03 & 3.35 & 16.7 & -235 \\
\hline Methane & $\mathrm{CH}_{4}$ & 16.04 & 6.04 & 46.0 & -82.6 \\
\hline Ammonia & $\mathrm{NH}_{3}$ & 17.03 & 7.33 & 113 & 132 \\
\hline Water & $\mathrm{H}_{2} \mathrm{O}$ & 18.02 & 6.87 & 221 & 374 \\
\hline Heavy water & $\mathrm{D}_{2} \mathrm{O}$ & 20.03 & 7.52 & 217 & 371 \\
\hline Neon & $\mathrm{Ne}$ & 20.18 & 3.00 & 26.8 & -229 \\
\hline Carbon monoxide & $\mathrm{CO}$ & 28.01 & 5.00 & 34.9 & -140 \\
\hline Nitrogen & $\mathrm{N}_{2}$ & 28.01 & 5.00 & 34.0 & -147 \\
\hline Ethylene & $\mathrm{C}_{2} \mathrm{H}_{4}$ & 28.05 & 7.95 & 50.4 & 9.2 \\
\hline Ethane & $\mathrm{C}_{2} \mathrm{H}_{6}$ & 30.07 & 10.8 & 48.7 & 32.2 \\
\hline Oxygen & $\mathrm{O}_{2}$ & 32.00 & 5.00 & 50.4 & -119 \\
\hline Methanol & $\mathrm{CH}_{3} \mathrm{OH}$ & 32.04 & 12.6 & 82.2 & 240 \\
\hline Fluoromethane & $\mathrm{CH}_{3} \mathrm{~F}$ & 34.03 & 7.27 & 59.0 & 44.1 \\
\hline Hydrogen sulfide & $\mathrm{H}_{2} \mathrm{~S}$ & 34.08 & 6.45 & 90.0 & 100 \\
\hline Fluorine & $\mathrm{F}_{2}$ & 38.00 & 5.03 & 51.7 & -129 \\
\hline Argon & $\mathrm{Ar}$ & 39.95 & 3.00 & 48.6 & -122 \\
\hline Propyne & $\mathrm{C}_{3} \mathrm{H}_{4}$ & 40.06 & 15.5 & 56.3 & 129 \\
\hline Propylene & $\mathrm{C}_{3} \mathrm{H}_{6}$ & 42.08 & 16.0 & 45.6 & 91.1 \\
\hline Cyclopropane & $\mathrm{C}_{3} \mathrm{H}_{6}$ & 42.08 & 16.4 & 55.8 & 125 \\
\hline Carbon dioxide & $\mathrm{CO}_{2}$ & 44.01 & 7.00 & 73.8 & 31.0 \\
\hline Nitrous oxide & $\mathrm{N}_{2} \mathrm{O}$ & 44.01 & 7.40 & 72.5 & 36.4 \\
\hline Propane & $\mathrm{C}_{3} \mathrm{H}_{8}$ & 44.10 & 19.1 & 42.5 & 96.7 \\
\hline Dimethylether & $\mathrm{C}_{2} \mathrm{H}_{6} \mathrm{O}$ & 46.07 & 17.2 & 53.4 & 127 \\
\hline Ethanol & $\mathrm{CH}_{3} \mathrm{CH}_{2} \mathrm{OH}$ & 46.07 & 22.6 & 61.5 & 241 \\
\hline Difluoromethane & $\mathrm{CH}_{2} \mathrm{~F}_{2}$ & 52.02 & 9.36 & 57.8 & 78.1 \\
\hline Butene & $\mathrm{C}_{4} \mathrm{H}_{8}$ & 56.11 & 24.9 & 40.1 & 146 \\
\hline Cis-butene & $c-\mathrm{C}_{4} \mathrm{H}_{8}$ & 56.11 & 24.6 & 42.3 & 163 \\
\hline Isobutene & $i-\mathrm{C}_{4} \mathrm{H}_{8}$ & 56.11 & 25.6 & 40.1 & 145 \\
\hline Trans-butene & $t-\mathrm{C}_{4} \mathrm{H}_{8}$ & 56.11 & 25.6 & 40.3 & 155 \\
\hline Acetone & $\mathrm{CH}_{3} \mathrm{COCH}_{3}$ & 58.08 & 24.3 & 47.0 & 235 \\
\hline$n$-butane & $\mathrm{C}_{4} \mathrm{H}_{10}$ & 58.12 & 29.5 & 38.0 & 152 \\
\hline Isobutane & $i-\mathrm{C}_{4} \mathrm{H}_{10}$ & 58.12 & 28.4 & 36.3 & 135 \\
\hline Carbon oxide sulfide & $\mathrm{COS}$ & 60.08 & 8.84 & 63.7 & 106 \\
\hline Sulfur dioxide & $\mathrm{SO}_{2}$ & 64.06 & 8.70 & 78.8 & 157 \\
\hline R152a & $\mathrm{CHF}_{2} \mathrm{CH}_{3}$ & 66.05 & 17.5 & 45.2 & 113 \\
\hline Trifluoromethane & $\mathrm{CHF}_{3}$ & 70.01 & 10.3 & 48.3 & 26.1 \\
\hline Nitrogen trifluoride & $\mathrm{NF}_{3}$ & 71.02 & 9.21 & 44.6 & -39.2 \\
\hline Isopentane & $i-\mathrm{C}_{5} \mathrm{H}_{12}$ & 72.15 & 39.3 & 33.8 & 187 \\
\hline Neopentane & neo- $\mathrm{C}_{5} \mathrm{H}_{12}$ & 72.15 & 38.1 & 32.0 & 161 \\
\hline Pentane & $\mathrm{C}_{5} \mathrm{H}_{12}$ & 72.15 & 39.8 & 33.7 & 197 \\
\hline Benzene & $\mathrm{C}_{6} \mathrm{H}_{6}$ & 78.11 & 34.2 & 48.9 & 289 \\
\hline Krypton & $\mathrm{Kr}$ & 83.80 & 3.00 & 55.3 & -63.7 \\
\hline R143a & $\mathrm{CF}_{3} \mathrm{CH}_{3}$ & 84.04 & 18.7 & 37.6 & 72.7 \\
\hline Cyclohexane & $\mathrm{C}_{6} \mathrm{H}_{12}$ & 84.16 & 48.4 & 40.8 & 280 \\
\hline Hexane & $\mathrm{C}_{6} \mathrm{H}_{14}$ & 86.18 & 50.9 & 30.3 & 235 \\
\hline Isohexane & $i-\mathrm{C}_{6} \mathrm{H}_{14}$ & 86.18 & 50.7 & 30.4 & 225 \\
\hline R22 & $\mathrm{CHClF}_{2}$ & 86.47 & 13.2 & 49.9 & 96.1 \\
\hline R14 & $\mathrm{CF}_{4}$ & 88.01 & 10.3 & 37.5 & -45.6 \\
\hline Toluene & $\mathrm{C}_{7} \mathrm{H}_{8}$ & 92.14 & 44.8 & 41.3 & 319 \\
\hline
\end{tabular}


TABLE I. (Continued.)

\begin{tabular}{|c|c|c|c|c|c|}
\hline Name & Chemical formula & $\begin{array}{c}M \\
(\mathrm{~g} / \mathrm{mol})\end{array}$ & $N(-)$ & $\begin{array}{c}P_{c} \\
\text { (bar) }\end{array}$ & $\begin{array}{c}T_{c} \\
\left({ }^{\circ} \mathrm{C}\right)\end{array}$ \\
\hline Heptane & $\mathrm{C}_{7} \mathrm{H}_{16}$ & 100.2 & 62.2 & 27.4 & 267 \\
\hline $\mathrm{R} 142 \mathrm{~b}$ & $\mathrm{C}_{2} \mathrm{H}_{3} \mathrm{ClF}_{2}$ & 100.5 & 22.3 & 40.6 & 137 \\
\hline $\mathrm{R} 134 \mathrm{a}$ & $\mathrm{CH}_{2} \mathrm{FCF}_{3}$ & 102.0 & 21.6 & 40.6 & 101 \\
\hline $\mathrm{R} 21$ & $\mathrm{CHCl}_{2} \mathrm{~F}$ & 102.9 & 15.8 & 51.8 & 178 \\
\hline R13 & $\mathrm{CClF}_{3}$ & 104.5 & 14.1 & 38.8 & 28.9 \\
\hline Octane & $\mathrm{C}_{8} \mathrm{H}_{18}$ & 114.2 & 73.8 & 25.0 & 296 \\
\hline $\mathrm{R} 141 \mathrm{~b}$ & $\mathrm{C}_{2} \mathrm{H}_{3} \mathrm{Cl}_{2} \mathrm{~F}$ & 116.9 & 25.4 & 42.1 & 204 \\
\hline R125 & $\mathrm{HF}_{2} \mathrm{C}-\mathrm{CF}_{3}$ & 120.0 & 22.5 & 36.2 & 66.0 \\
\hline R12 & $\mathrm{CCl}_{2} \mathrm{~F}_{2}$ & 120.9 & 17.7 & 41.4 & 112 \\
\hline Nonane & $\mathrm{C}_{9} \mathrm{H}_{20}$ & 128.3 & 85.6 & 22.8 & 321 \\
\hline Xenon & $\mathrm{Xe}$ & 131.3 & 3.00 & 58.4 & 16.6 \\
\hline $\mathrm{R} 245 \mathrm{ca}$ & $\mathrm{CHF}_{2} \mathrm{CF}_{2} \mathrm{CH}_{2} \mathrm{~F}$ & 134.0 & 35.4 & 39.3 & 174 \\
\hline R245fa & $\mathrm{CF}_{3} \mathrm{CH}_{2} \mathrm{CHF}_{2}$ & 134.0 & 32.9 & 36.5 & 154 \\
\hline R124 & $\mathrm{CHClFCF}_{3}$ & 136.5 & 26.1 & 36.2 & 122 \\
\hline R11 & $\mathrm{CCl}_{3} \mathrm{~F}$ & 137.4 & 20.2 & 44.1 & 198 \\
\hline R116 & $\mathrm{CF}_{3} \mathrm{CF}_{3}$ & 138.0 & 23.3 & 30.5 & 19.9 \\
\hline Decane & $\mathrm{C}_{10} \mathrm{H}_{22}$ & 142.3 & 97.5 & 21.0 & 345 \\
\hline Sulfur hexafluoride & $\mathrm{SF}_{6}$ & 146.1 & 22.5 & 37.5 & 45.6 \\
\hline R236ea & $\mathrm{CF}_{3} \mathrm{CHFCHF}_{2}$ & 152.0 & 35.1 & 35.0 & 139 \\
\hline R236fa & $\mathrm{CF}_{3} \mathrm{CH}_{2} \mathrm{CF}_{3}$ & 152.0 & 34.0 & 32.0 & 125 \\
\hline R123 & $\mathrm{CHCl}_{2} \mathrm{CF}_{3}$ & 152.9 & 28.6 & 36.6 & 184 \\
\hline R115 & $\mathrm{CClF}_{2} \mathrm{CF}_{3}$ & 154.5 & 27.1 & 31.3 & 80.0 \\
\hline MM & $\mathrm{C}_{8} \mathrm{H}_{18} \mathrm{OSi}_{2}$ & 162.4 & 77.4 & 19.4 & 246 \\
\hline R227ea & $\mathrm{CF}_{3} \mathrm{CHFCF}_{3}$ & 170.0 & 35.4 & 29.3 & 102 \\
\hline Dodecane & $\mathrm{C}_{12} \mathrm{H}_{26}$ & 170.3 & 122 & 18.2 & 385 \\
\hline R114 & $\mathrm{CClF}_{2} \mathrm{CClF}_{2}$ & 170.9 & 31.2 & 32.6 & 146 \\
\hline R113 & $\mathrm{CCl}_{2} \mathrm{FCClF}_{2}$ & 187.4 & 34.0 & 33.9 & 214 \\
\hline R218 & $\mathrm{CF}_{3} \mathrm{CF}_{2} \mathrm{CF}_{3}$ & 188.0 & 36.8 & 26.4 & 71.9 \\
\hline $\mathrm{RC} 318$ & $\mathrm{C}_{4} \mathrm{~F}_{8}$ & 200.0 & 41.9 & 27.8 & 115 \\
\hline MDM & $\mathrm{C}_{8} \mathrm{H}_{24} \mathrm{O}_{2} \mathrm{Si}_{3}$ & 236.5 & 115 & 14.2 & 291 \\
\hline $\mathrm{D}_{4}$ & $\mathrm{C}_{8} \mathrm{H}_{24} \mathrm{O}_{4} \mathrm{Si}_{4}$ & 296.6 & 130 & 13.3 & 313 \\
\hline $\mathrm{D}_{5}$ & $\mathrm{C}_{10} \mathrm{H}_{30} \mathrm{O}_{5} \mathrm{Si}_{5}$ & 370.8 & 172 & 11.6 & 346 \\
\hline $\mathrm{MD}_{4} \mathrm{M}$ & $\mathrm{C}_{14} \mathrm{H}_{42} \mathrm{O}_{5} \mathrm{Si}_{6}$ & 459.0 & 235 & 8.77 & 380 \\
\hline
\end{tabular}

ever, differently from the ideal-gas case, the behavior of the dimensionless flow variables in dense-gas expansions is dependent on the reduced inlet conditions, cf. Eqs. (13) and (14). Therefore, it is not possible to repeat the extended analysis performed for the ideal-gas case, see Figs. 4-6, also for the dense-gas case. Here, only three different reduced inlet conditions are considered to evaluate the influence of the molecular complexity $N$ on the exit Mach number, the speed of sound and the velocity.

The inlet conditions of the three expansion processes are given in reduced terms in Table II. In this table, the compressibility factor $Z \equiv P v /(R T)$ quantifies the degree of thermodynamic nonideality, i.e., the deviation of volumetric properties from ideal-gas behavior, for which $Z \equiv 1$. These processes represent dense-gas expansions starting from state point of increasing nonideality, as set into evidence by their respective inlet compressibility factors of $Z_{1}=0.9,0.8$, and 0.7 .

Results for the three initial conditions in Table II are shown in Figs. 8-10, where three different expansion ratios are also considered in order to investigate the dependence on this parameter. PVdW results are plotted together with idealgas results and with those obtained using highly accurate multiparameter EoSs of the 75 fluids listed in Table I.

The qualitative behavior of dense-gas expansions is similar to the one observed in ideal-gas expansions possibly because the chosen inlet conditions, which are representative for industrial applications, ${ }^{36}$ are not far from the ideal-gas region. This includes the change in monotonicity in the speed of sound at a given expansion ratio that results in the nonmonotone behavior of the Mach number with respect to molecular complexity. For dense gases, this behavior depends on the reduced thermodynamic state, as well as on the molecular complexity and expansion ratio as discussed in the ideal-gas case. Quantitative differences between the polytropic ideal and the PVdW models are limited to about $25 \%$ for the speed of sound and the flow velocity, and to 5\% for the Mach number and are most relevant for the lowest pres- 


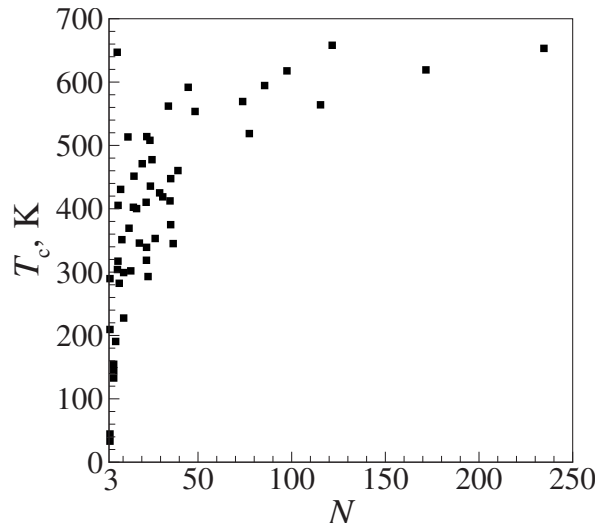

FIG. 7. Critical temperature vs the number of active degrees of freedom for selected fluids listed in Table I.

sure ratio, for which the outlet state is closest to the initial, nonideal, one. The difference in sound speed reduces at higher pressure ratios since these expansions have outlet states which are located well into the ideal-gas region $\left(Z_{2}=1\right)$. The PIG model does not account for intermolecular forces and hence overpredicts both sound speed and velocity. ${ }^{15}$ Sound speed and velocity are overpredicted to a different extent so that as a net result the Mach number, defined as $\mathrm{Ma}_{2}=u_{2} / c_{2}$, is underpredicted by the PIG model. In dense-gas expansions, the contribution of the repulsive molecular forces in the dense-gas region leads to a lower sound speed, as shown in Ref. 15, and due to a lower specific enthalpy difference, to a lower flow velocity than what would be expected assuming an ideal gas. It is remarkable that the prediction from the very accurate MPEoS is indistinguishable from the Van der Waals model, within $1 \%$ in the worst cases. As anticipated, the MPEoSs implicitly takes into account additional parameters which allows for the molecular complexity of the fluid to include others characteristics such as Coulomb, dipole-dipole, three, and many body potentials. It is therefore confirmed here that the most relevant parameter which determines the dense-gas behavior of a given fluid is its molecular complexity defined simply in terms of active degrees of freedom $N$, as observed also in Ref. 14, using very simple models. Moreover, the fact that the Van der Waals and the MPEoS results are almost coincident if expressed in reduced variables points to the fact that the law of corresponding states can be used to compute dense-gas expansions accurately, at least in the mildly nonideal conditions considered here.

To conclude, it should be noted that only postexpansion fluid dynamic quantities are considered here. The qualitative trends in the sound speed, velocity, and Mach number along

TABLE II. Inlet conditions for the three dense-gas expansions in terms of reduced values.

\begin{tabular}{lcccc}
\hline \hline Reduced total inlet pressure & $P_{1}^{t} / P_{c}$ & 0.4 & 0.7 & 1 \\
Reduced total inlet temperature & $T_{1}^{t} / T_{c}$ & 1.1 & 1.1 & 1.1 \\
Compressibility factor at the inlet & $Z_{1}$ & 0.9 & 0.8 & 0.7 \\
\hline \hline
\end{tabular}
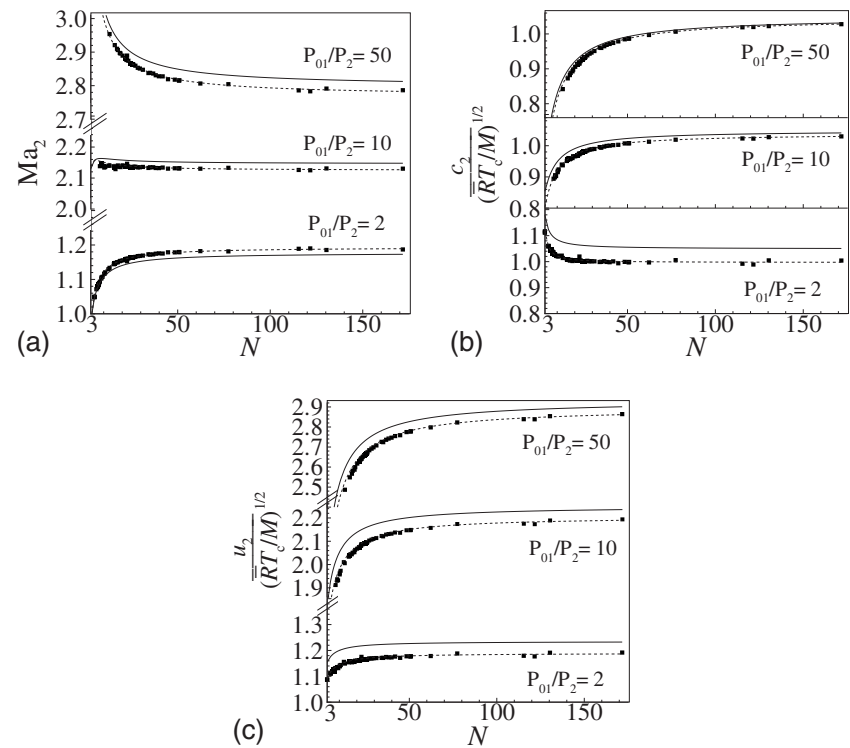

FIG. 8. Fluid dynamic quantities at outlet for fluids of increasing complexity listed in Table I at pressure ratios $P_{1}^{t} / P_{2}=2,10$, and 50. Inlet conditions are given in Table II, for an inlet compressibility factor $Z_{1}=0.9$. Results are given for the PIG model (-), PVdW model (- - -), and the MPEoSs of various fluids ( $\mathbf{\square})$. Results of the MPEoS models for which condensation occurs have been omitted.

the expansion is possibly very different for complex fluids compared to that of simple molecules, as discussed in Sec. IV.

\section{2D FLOW SIMULATIONS OF EXPANDING FLOWS}

The flow through the stator of an exemplary turbine cascade has been simulated as an application of the findings of Sec. III B. The cascade is formed by two VKI LS-89 turbine
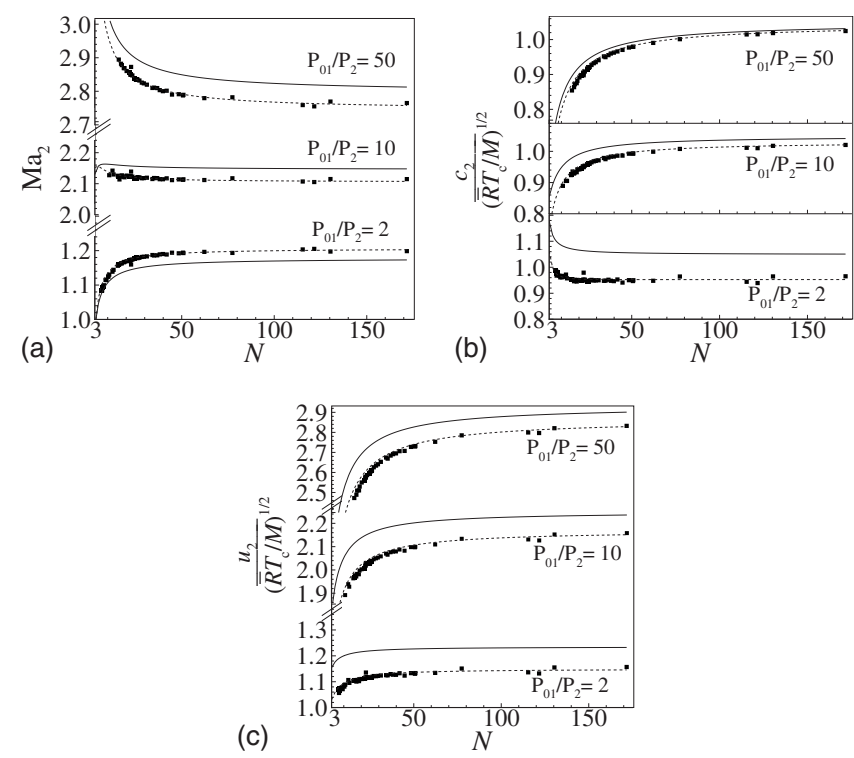

FIG. 9. Fluid dynamic quantities at outlet for fluids of increasing complexity listed in Table I at pressure ratios $P_{1}^{t} / P_{2}=2,10$, and 50. Inlet conditions are given in Table II, for an inlet compressibility factor $Z_{1}=0.8$. Results are given for the PIG model (-), PVdW model (- - -), and the MPEoSs of various fluids (ם). Results of the MPEoS models for which condensation occurs have been omitted. 

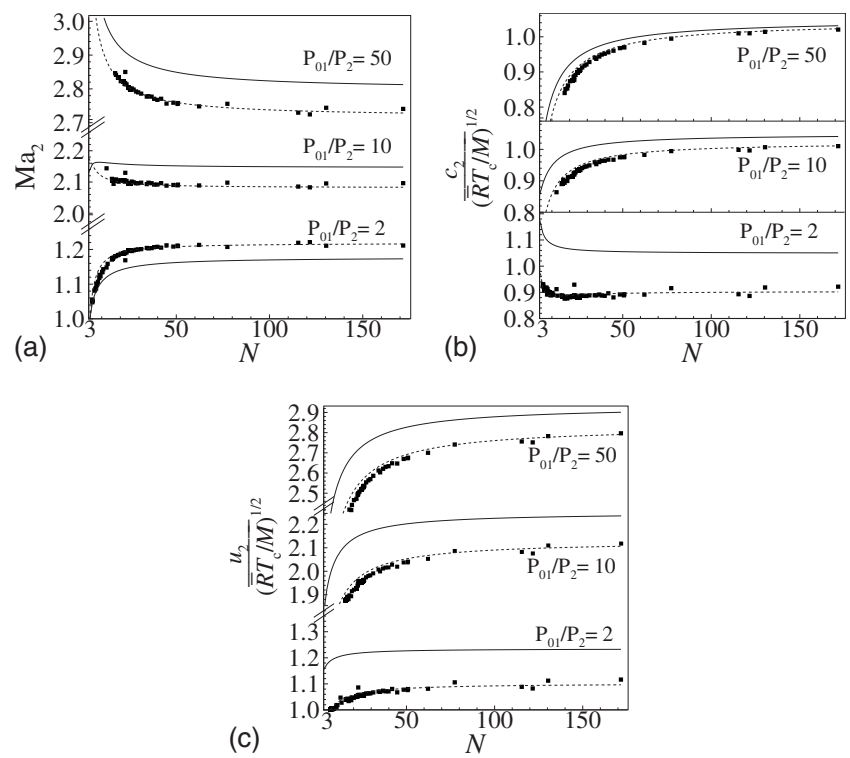

FIG. 10. Fluid dynamic quantities at outlet for fluids of increasing complexity listed in Table I at pressure ratios $P_{1}^{t} / P_{2}=2,10$, and 50. Inlet conditions are given in Table II, for an inlet compressibility factor $Z_{1}=0.7$. Results are given for the PIG model (-), PVdW model (- - ), and the MPEoSs of various fluids (ם). Results of the MPEoS models for which condensation occurs have been omitted.

blades ${ }^{37}$ which is a standard high pressure turbine nozzle guide vane. It should be noted that in reality the fluid dynamic design of the stator would lead to a different blade profile, depending on the fluid and operating conditions. Three industrial working fluids for power and refrigeration cycles are considered, namely, steam, carbon dioxide, and propane. Example of applications are power plants (steam), particle separation processes $\left(\mathrm{CO}_{2}\right)$, and refrigeration (propane).

Steam, carbon dioxide, and propane belong to different so-called fluid families because they have different molecular structures and are made of different atomic species. These fluids are chosen here because they are conveniently different in molecular complexity and weight. Their molecular weight and complexity, in terms of both the number of active molecular degrees of freedom, $N$, and the minimum value of the fundamental derivative along the saturation line, $\Gamma_{\min }^{V}$, are given in Table III. As it is evident from the table, steam and carbon dioxide have very similar molecular complexities but due to their different molecular structures and atoms, they have very different molecular weights. Similarly, carbon dioxide and propane have nearly the same molecular weight but propane is much more complex. The inlet conditions for the simulated dense-gas expansions are taken from the moderate $\left(Z_{1}=0.8\right)$ dense-gas expansion defined in Sec. III B and are specified in Table II in terms of reduced variables. In this case a pressure ratio of $P_{1}^{t} / P_{2}=1.94$ is used. The dimensional process conditions are also listed in Table III. The compressibility factor indicates that, even though the expansions occur in the dense-gas region, they occur quite close to the region of gas ideality.

For each fluid, a Mach-density plot is given in Fig. 11, which shows a monotonous increase in the Mach number
TABLE III. Parameters characterizing the expansion processes of steam, carbon dioxide, and propane. The expansions correspond to the moderate $\left(Z_{1}=0.8\right)$ dense-gas expansion defined in Sec. III B, as specified in reduced conditions in Table II.

\begin{tabular}{|c|c|c|c|c|}
\hline & & Steam & Carbon dioxide & Propane \\
\hline Chemical formula & & $\mathrm{H}_{2} \mathrm{O}$ & $\mathrm{CO}_{2}$ & $\mathrm{C}_{3} \mathrm{H}_{8}$ \\
\hline Molecular weight & $M, \mathrm{~g} / \mathrm{mol}$ & 18.02 & 44.01 & 44.1 \\
\hline $\begin{array}{l}\text { Fundamental derivative } \\
\text { of gasdynamics }\end{array}$ & $\Gamma_{\min }^{V},-$ & 1.162 & 1.257 & 0.864 \\
\hline $\begin{array}{l}\text { Number of active degrees } \\
\text { of freedom }\end{array}$ & $N,-$ & 6.9 & 7.0 & 19.1 \\
\hline Critical pressure & $P_{c}$, bar & 220.6 & 73.8 & 42.5 \\
\hline Critical temperature & $T_{c},{ }^{\circ} \mathrm{C}$ & 374 & 32 & 96.7 \\
\hline MPEoS & & a & b & $\mathrm{c}$ \\
\hline Total inlet pressure & $P_{1}^{t}$, bar & 154.4 & 51.6 & 29.76 \\
\hline Total inlet temperature & $T_{1}^{t},{ }^{\circ} \mathrm{C}$ & 438.7 & 61.4 & 133.7 \\
\hline $\begin{array}{l}\text { Compressibility factor } \\
\text { at inlet }\end{array}$ & $Z_{1},-$ & 0.81 & 0.8 & 0.8 \\
\hline Static outlet pressure & $P_{2}$, bar & 79.7 & 26.6 & 15.4 \\
\hline $\begin{array}{l}\text { Compressibility factor } \\
\text { at outlet }\end{array}$ & $Z_{2},-$ & 0.82 & 0.82 & 0.87 \\
\hline
\end{tabular}

${ }^{\mathrm{a}}$ Reference 9 .

${ }^{\mathrm{b}}$ Reference 38 .

${ }^{\mathrm{c}}$ Reference 39.

with decreasing density. Only HMC fluids may possibly feature a nonmonotonous Mach number variation with density in a limited thermodynamic region. ${ }^{25,26}$ This is not the case for the thermodynamic conditions considered here.

The ZFLOW code $^{40}$ is used to simulate the inviscid 2D flow field generated by the expansion of the three fluids through the passage formed by two blades. To take into account thermodynamic nonideality and molecular influences additional to the number of active molecular degrees of free-
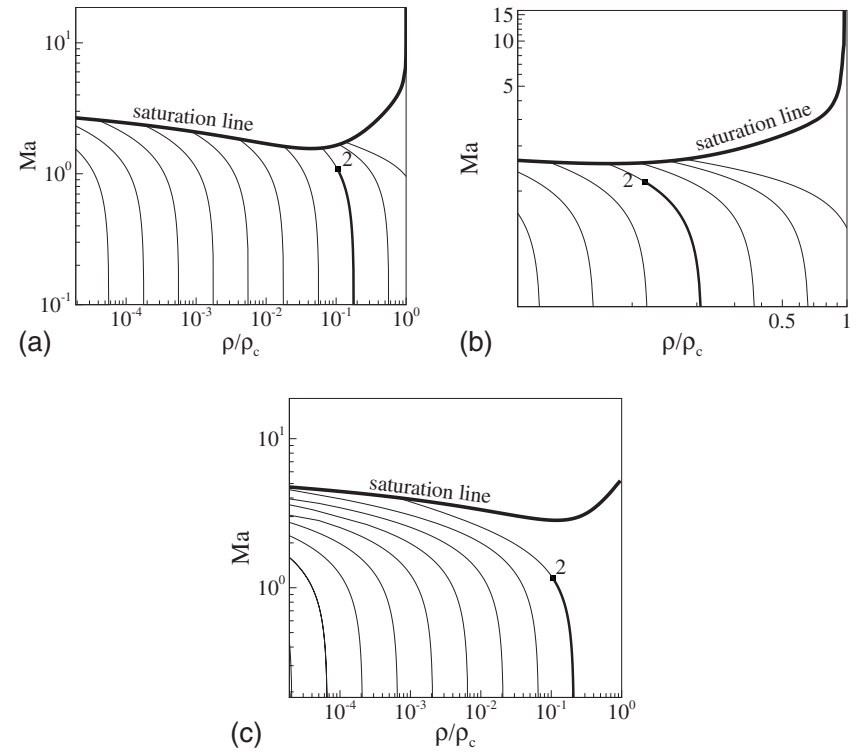

FIG. 11. Variation of Mach number with density for steam (a), carbon dioxide (b), and propane (c) along expansion isentropes starting from the inlet total pressure $P_{1}^{t} / P_{c}=0.7$, which is equal to the inlet state of the expansion with $Z_{1}=0.8$ given in Table II. Thick lines indicate saturation curves; thin ones isentropes. Initial or total conditions are at $\mathrm{Ma}=0$. 
(a)

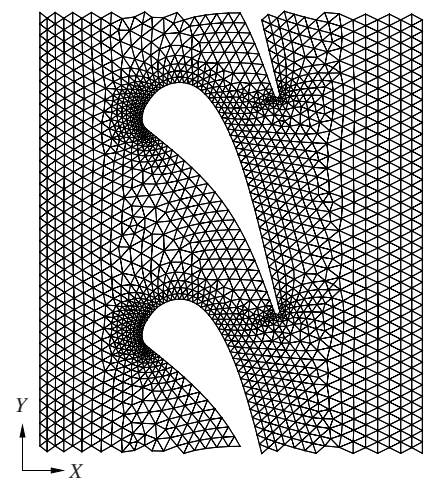

(b)

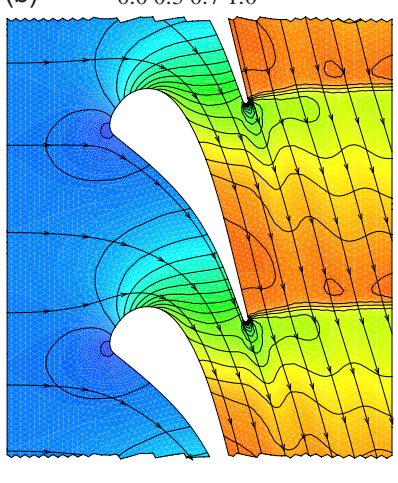

FIG. 12. (Color online) (a) Coarse grid (1302 nodes) of the VKI LS-89 blade. $X$ denotes the axial coordinate of the nozzle and $Y$ denotes the nozzle ring circumferential coordinate. Periodicity allowed the computations to be performed using only the top half of the grid depicted here. (b) Mach number flow field for steam and the expansion conditions given in Table III.

dom, the MPEoSs of water, ${ }^{9}$ carbon dioxide, ${ }^{38}$ and propane ${ }^{39}$ of the respective fluids are used to evaluate the thermodynamic properties. All flow solutions have converged up to at least a reduction of five orders of magnitude, with respect to the first iteration, measured in the $L^{2}$-norm of the conserved variables. To ensure a small discretization error, a grid convergence study has been performed by computing the solution on three grids with decreasing element size. ${ }^{11}$ The coarse grid is shown in Fig. 12, together with the Mach number field and streamlines obtained on the finer grid for steam. The finest grid used in the computation is made of 4153 nodes and 6939 triangles.

The blade surface distributions of relevant flow quantities obtained from the simulation on the fine grid are depicted in Figs. 13 and 14. Surface distributions are plotted as a function of the reduced coordinate $X / C_{\mathrm{ax}}$, where $X$ is the axial coordinate and $C_{\mathrm{ax}}$ is the axial blade chord.

The distribution of the fundamental derivative of gas dynamics in Fig. 13(a) indicates that for the chosen expansion conditions, steam and carbon dioxide both exhibit LMC behavior $(\Gamma>1)$ whereas propane behaves as a HMC fluid $(\Gamma<1)$.

Figure 13(b) shows that the sound speed in steam is almost twice as high as that of carbon dioxide and propane, as they have a higher molecular weight. The dimensional sound speed is strongly influenced by molecular weight. More importantly, the small difference between the sound speeds in carbon dioxide and propane, which have almost the same molecular weight, is the result of the weak influence of molecular complexity on the speed of sound, as explained in Sec. III. By nondimensionalizing the sound speed with the reference velocity $\sqrt{\bar{R}} T_{c} / M$, which is different for each of the three fluids, the strong contribution of the molecular weight is eliminated. The HMC behavior of propane is clearly visible in Fig. 13(c), which shows that the speed of sound increases along the expansion. The quantitative differences in Fig. 13(c) are solely due to the weak influence of molecular complexity, as was observed in Fig. 9(b).

The variation of the Mach number with density is quan-
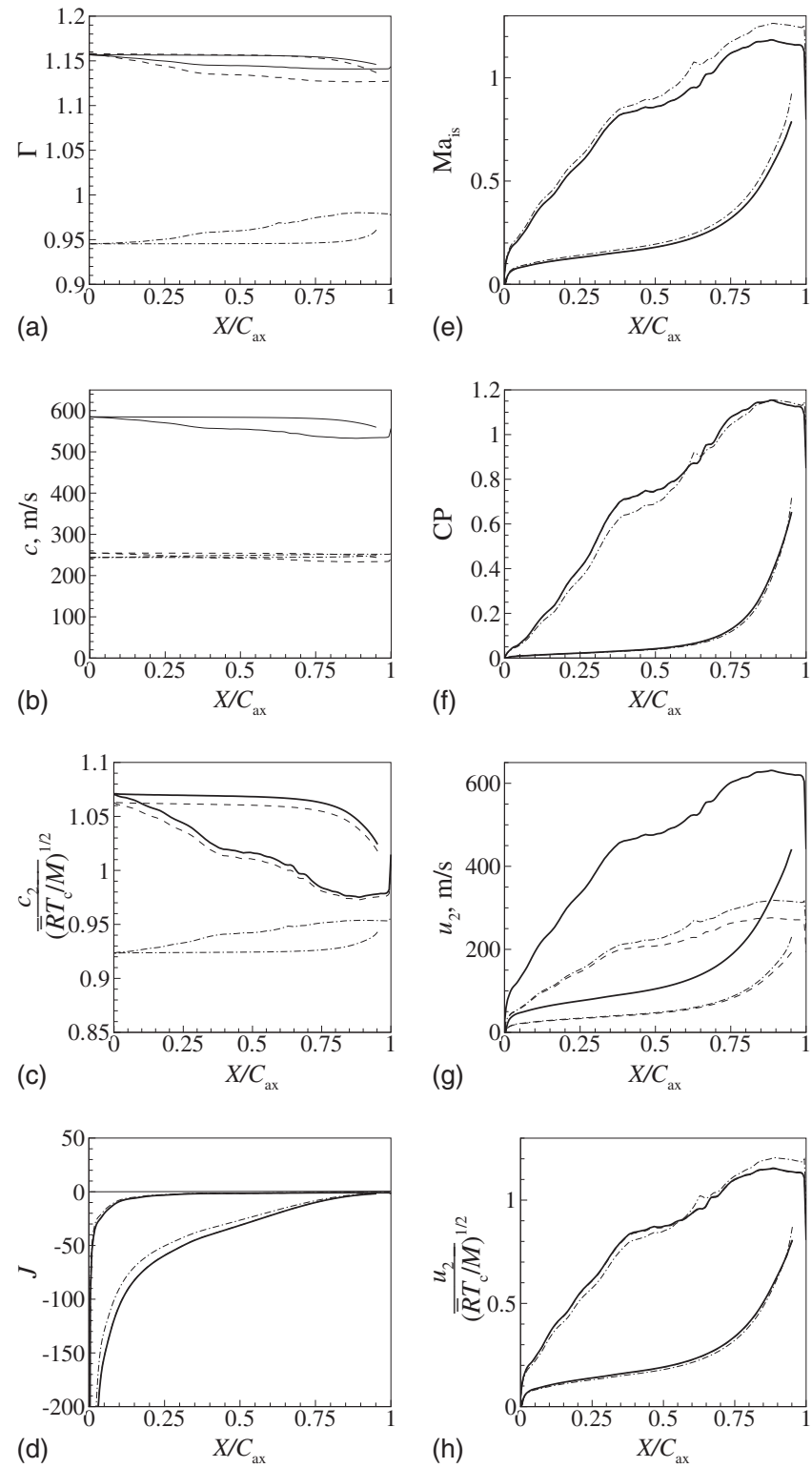

FIG. 13. Blade surface distributions of the fundamental derivative of gasdynamics (a), sound speed (b), dimensionless sound speed (c), $J=1-\Gamma$ $-1 / \mathrm{Ma}^{2}$ (d), Mach number (e), pressure coefficient (f), velocity (g), and dimensionless velocity (h) for steam (-), carbon dioxide (- - -), and propane $(-\cdot-\cdot-)$.

tified by the variable $J=1-\Gamma-1 / \mathrm{Ma}^{2}$ - see, e.g., Ref. 26 . The Mach number decreases with decreasing density (i.e., for an expansion) only if $J>0$. For $J<0$, the Mach number increases with decreasing density, which is the case for the three expanded fluids and for the considered conditions, as shown in Fig. 13(d).

The Mach number distribution itself, plotted in Fig. 13(e), is identical for steam and carbon dioxide which have molecules of similar complexity, and is not influenced by their different molecular weights and critical temperatures, as expected based on the observations made in Sec. III. For the same pressure ratio, the more complex fluid propane achieves significantly higher Mach numbers.

The pressure coefficient along the blade, which is defined as $\mathrm{CP}=\left(P_{1}^{t}-P\right) /\left(P_{1}^{t}-P_{2}\right)$ and exhibits no relevant dif- 

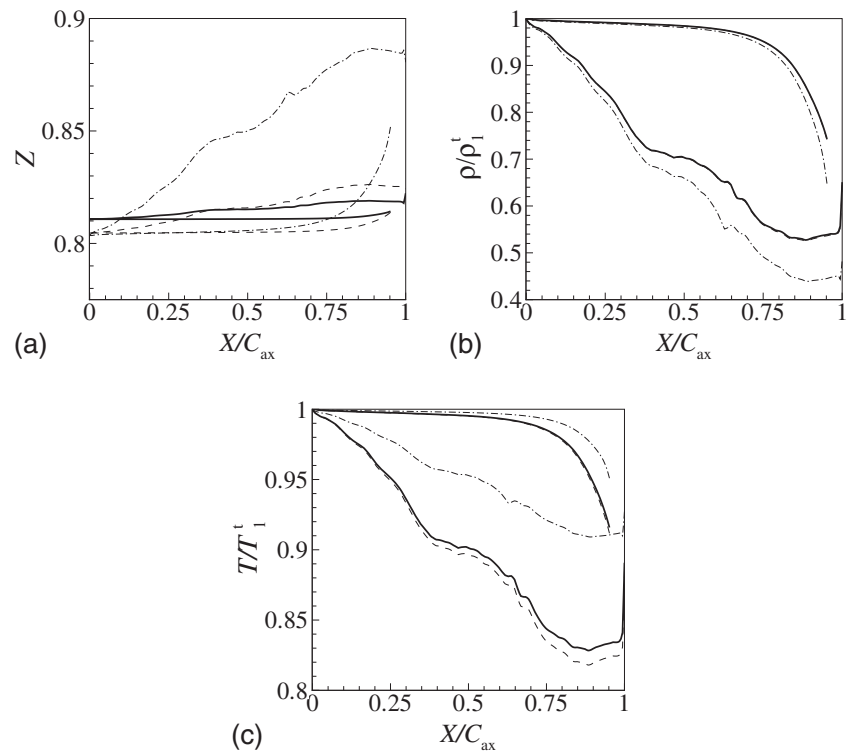

FIG. 14. Blade surface distributions of compressibility factor (a), density (b), and temperature (c) for steam (-), carbon dioxide (- - -), and propane $(-\cdot-\cdot-)$.

ferences for the three fluids, is reported in Fig. 13(f). Note that the pressure ratio is the same for the three fluids and hence only minor differences in the pressure coefficient along the blade can be expected.

Similarly to the speed of sound, the flow velocity, depicted in Fig. 13(g), is strongly influenced by molecular weight. The expansion of steam leads to velocities more than a factor of 2 higher than those obtained for the much heavier carbon dioxide and propane. However, the difference between these two fluids having nearly the same molecular weight is also appreciable. Since the dimensionless velocity in the flows of carbon dioxide and propane are almost coincident, see Fig. 13(h), the differences in the dimensional velocity is believed to be related to the different critical temperature of the two fluids, see Sec. III. The differences in the dimensionless velocities at the outflow are coherent with the results shown in Fig. 9(c), in which the velocity only slightly varies for $P_{1}^{t} / P_{2}=2$, even for low values of $N$.

Figure 14(a) reports the compressibility factor $Z$ along the blade. As expected, $Z$ is always close to one for all fluids and hence the considered expansions are only mildly nonideal. The fluids are expanded from the same inlet state in reduced terms, and hence, following the principle of corresponding states, their compressibility factors are very similar. The small deviation is due to the fact that the principle of corresponding states cannot take into account influences other than the critical pressure and temperature on the volumetric behavior. As is evident from Fig. 14(a), the expansion of the more complex fluid propane ends closer to its idealgas region than those of steam and carbon dioxide. This becomes clear when considering the temperature.

The absolute value of the density is very different for the three fluids because it is determined by the critical density $\rho_{c}$. However, the qualitative behavior of the density, shown in Fig. 14(b) in terms of the density reduced with respect to the inlet value, is near identical for the similarly complex fluids steam and carbon dioxide. The more complex propane has a different distribution because for the same pressure ratio, the expansion of a more complex fluid leads to lower reduced densities. Finally, the qualitative behavior of the temperature, given in Fig. 14(c) in terms of the temperature reduced by the inlet value, is also similar for steam and carbon dioxide. As discussed in Sec. III A, for complex fluids, the outlet temperature is closer to the inlet value. Conversely, LMC fluids such as steam and carbon dioxide exhibit a fairly large reduced temperature drop. The expansion of the more complex propane leads to only half of this reduced temperature drop.

\section{CONCLUSIONS}

This paper presents an investigation on the effect of the complexity of a fluid molecule on the fluid dynamic quantities sound speed, velocity, and Mach number in ideal and mildly nonideal isentropic expansions. The influence of molecular complexity on expanding flows is investigated for ideal-gas and dense-gas expansions, respectively. The analysis is carried out using the number of active degrees of freedom as a measure of molecular complexity, in combination with the PIG and Van der Waals gas model. These results are then verified using highly accurate MPEoSs.

For isentropic expansions, the Mach number does not depend on the molecular weight of the fluid but only on molecular complexity and pressure ratio. Remarkably enough, the Mach number can either increase or decrease with molecular complexity, depending on the considered pressure ratio. More precisely, for low pressure ratios, the Mach number is a monotonically increasing function of molecular complexity. For higher pressure ratios, the Mach number first sharply increases to a maximum (at molecular complexities approximately corresponding to monatomic fluids) and then decreases for fluids of increasing molecular complexity. The exit speed of sound and flow velocity, however, are dependent on both molecular complexity and weight, as well as on the inlet total temperature. The exit flow velocity is found to be a monotonically increasing function of molecular complexity regardless of the expansion ratio, whereas the speed of sound increases monotonically with molecular complexity for high pressure ratios. Nonmonotone behavior of the speed of sound exists for pressure ratios around 3, which leads to similarly nonmonotone behavior of the Mach number at pressure ratios around 10. It is important to note, however, that sound speed and flow velocity depend much more strongly on molecular weight than on molecular complexity, which in realistic expansions, often obscures the influence of the latter. The results confirm that the most relevant parameter which determines the dense-gas behavior of a given fluid is its molecular complexity defined simply in terms of the number of active degrees of freedom.

Different from the ideal-gas case, in dense-gas expansions the behavior of the dimensionless flow variables sound speed and flow velocity is dependent on the reduced inlet conditions. Deviation from gas ideality affects the change in monotonicity in the exit speed of sound and exit Mach number at a given expansion ratio. For the dense-gas expansions 
considered here, the quantitative deviations from the idealgas case include a lower sound speed and, due to a lower specific enthalpy difference, a lower flow velocity.

The distinction made here between the influence of molecular complexity and weight helps to explain the difficulties in determining the correct similitude criteria in high Reynolds number transonic wind tunnels employing heavy gases as the working fluid. ${ }^{4-6}$ Thanks to its relatively high molecular weight, which is roughly six times that of air, sulfur hexafluoride is used in existing wind tunnels to increase the flow Reynolds number. However, due to the different molecular complexities $\left(N \simeq 5\right.$ for air and $N \simeq 20$ for $\mathrm{SF}_{6}$ at ambient temperature), the Mach number of the flow leaving the nozzle and entering the test chamber is different for the two fluids. In Ref. 6 , a correction is proposed in which the similitude parameter is obtained using a value of $\gamma$ that is computed from the inlet value of $\Gamma$, namely, the fluid molecular complexity.

The outcome of this investigation is also applicable in the design of turbines for organic Rankine cycle (ORC) turbogenerators. These power plants are suitable for the conversion of an external heat source into electricity in the lowpower range (few kilowatts up to few megawatts), by virtue of an organic working fluid, whose molecule is both complex and heavy. The results of this study show that the low rotational speed typical of ORC turbines is made possible by the high molecular weight of the organic working fluid, not by its complexity. For instance, assume that a cycle analysis yields two suitable organic working fluids that have similar molecular complexity but different molecular weights. The Mach number per turbine stage is limited, to avoid the occurrence of supersonic flow and associated shock losses in the rotor. The findings of this paper show that the heavier organic substance leads to lower nozzle expansion velocities, thus allowing for lower rotational speeds of the turbine. This, together with a higher volumetric flow rate, explains why heavy organic fluids are favorable for obtaining high turbine efficiencies.

\section{ACKNOWLEDGMENTS}

This research was supported by the Delft Centre for Sustainable Industrial Processes, the Dutch Technology Foundation STW, the Applied Science Division of NWO and the Technology Program of the Ministry of Economic Affairs, DSF 6573. The authors acknowledge the contribution of their colleague and friend Dr. Ryan Nannan for discussions on molecular complexity.

${ }^{1}$ G. Angelino, M. Gaia, and E. Macchi, "A review of Italian activity in the field of organic Rankine cycles," VDI Berichte_Proceedings of the International VDI Seminar (VDI Verlag, Düsseldorf, 1984), Vol. 539, pp. $465-482$.

${ }^{2}$ J. Larjola, "Electricity from industrial waste heat using high-speed organic Rankine cycle (ORC)," Int. J. Prod. Econ. 41, 227 (1995).

${ }^{3}$ G. Angelino and P. Colonna, "Multicomponent working fluids for organic Rankine Cycles (ORCs)," Energy 23, 449 (1998).

${ }^{4}$ J. J. Korte, "Inviscid design of hypersonic wind tunnel nozzles for a real gas," 38th Aerospace Sciences Meeting and Exhibit, Reno, NV, edited by E. Camhi (AIAA, Reston, 2000), pp. 1-8.

${ }^{5}$ W. K. Anderson, "Numerical study on using sulfur hexafluoride as a wind tunnel test gas," AIAA J. 29, 2179 (1991).
${ }^{6}$ J. B. Anders, W. K. Anderson, and A. V. Murthy, "Transonic similarity theory applied to a supercritical airfoil in heavy gases," J. Aircr. 36, 957 (1999).

${ }^{7}$ J. W. Tom and P. G. Debenedetti, "Particle formation with supercritical fluids—a review,” J. Aerosol Sci. 22, 555 (1991).

${ }^{8} \mathrm{M}$. Türk, "Influence of thermodynamic behaviour and solute properties on homogeneous nucleation in supercritical solutions," J. Supercrit. Fluids 18, 169 (2000).

${ }^{9}$ W. Wagner and A. Pruss, "The IAPWS formulation 1995 for the thermodynamic properties of ordinary water substance for general and scientific use," J. Phys. Chem. Ref. Data 31, 387 (2002).

${ }^{10}$ J. Hoffren, T. Talonpoika, J. Larjola, and T. Siikonen, "Numerical simulation of real-gas flow in a supersonic turbine nozzle ring," ASME J. Eng. Gas Turbines Power 124, 395 (2002).

${ }^{11}$ P. Colonna, J. Harinck, S. Rebay, and A. Guardone, "Real-gas effects in organic rankine cycle turbine nozzles," AIAA J. Propul. Power 24, 282 (2008).

${ }^{12}$ J. Harinck, P. Colonna, A. Guardone, and S. Rebay, "Influence of thermodynamic models in 2D flow simulations of turboexpanders," J. Turbomach. (in press).

${ }^{13}$ J. Harinck, T. Turunen-Saaresti, P. Colonna, and J. van Buijtenen, "Computational study of a high-expansion ratio radial ORC turbine stator," ASME J. Eng. Gas Turbines Power (in press).

${ }^{14}$ A. Guardone and B. M. Argrow, "Nonclassical gasdynamic region of selected fluorocarbons," Phys. Fluids 17, 116102 (2005).

${ }^{15} \mathrm{P}$. Colonna and A. Guardone, "Molecular interpretation of nonclassical gasdynamics of dense vapors under the Van der Waals model," Phys. Fluids 18, 056101 (2006).

${ }^{16}$ P. A. Thompson, "A fundamental derivative in gasdynamics," Phys. Fluids 14, 1843 (1971).

${ }^{17} \mathrm{H}$. A. Bethe, "On the theory of shock waves for an arbitrary equation of state," NTIS Document No. PB 032189, 1942 (Office for Science, Research and Development No. 545), U.S. Library of Congress, Washington, D.C. 20540.

${ }^{18} \mathrm{H}$. B. Callen, Thermodynamics and an Introduction to Thermostatistics, 2nd ed. (Wiley, New York, 1985).

${ }^{19}$ J. D. van der Waals, "On the continuity of the gas and liquid state," Ph.D. thesis, University of Leiden, 1873.

${ }^{20}$ R. Span, Multiparameter Equations of State: An Accurate Source of Thermodynamic Property Data (Springer-Verlag, Berlin, 2000).

${ }^{21}$ R. Span, W. Wagner, E. W. Lemmon, and R. T. Jacbosen, "Multiparameter equations of state-recent trends and future challenges," Fluid Phase Equilib. 183-184, 1 (2001).

${ }^{22}$ E. W. Lemmon and R. Span, "Short fundamental equations of state for 20 industrial fluids," J. Chem. Eng. Data 51, 785 (2006).

${ }^{23}$ E. W. Lemmon, M. O. McLinden, and M. L. Huber, REFProp: Reference Fluid Properties software, NIST, 2002.

${ }^{24} \mathrm{~W}$. Bober and W. L. Chow, "Nonideal isentropic gas flow through converging-diverging nozzles," ASME J. Fluids Eng. 112, 455 (1990).

${ }^{25}$ M. S. Cramer, "On the Mach number variation in steady flows of dense hydrocarbons," ASME J. Fluids Eng. 113, 675 (1991).

${ }^{26}$ M. S. Cramer and L. M. Best, "Steady, isentropic flows of dense gases," Phys. Fluids A 3, 219 (1991).

${ }^{27}$ M. S. Cramer and N. R. Fry, "Nozzle flows of dense gases," Phys. Fluids A 5, 1246 (1993).

${ }^{28}$ G. H. Schnerr and P. Leidner, "Diabatic supersonic flows of dense gases," Phys. Fluids A 3, 2445 (1991).

${ }^{29}$ A. Kluwick, "Internal flows of dense gases," Acta Mech. 169, 123 (2004).

${ }^{30}$ Ya. B. Zel'dovich, "On the possibility of rarefaction shock waves," Zh. Eksp. Teor. Fiz. 4, 363 (1946).

${ }^{31}$ P. A. Thompson and K. C. Lambrakis, "Negative shock waves," J. Fluid Mech. 60, 187 (1973)

${ }^{32}$ P. A. Thompson, Compressible Fluid Dynamics (McGraw-Hill, New York, 1988).

${ }^{33}$ R. Menikoff and B. J. Plohr, "The Riemann problem for fluid flow of real material," Rev. Mod. Phys. 61, 75 (1989).

${ }^{34}$ P. Colonna, A. Guardone, N. R. Nannan, and C. Zamfirescu, "Design of the dense gas flexible asymmetric shock tube," ASME J. Fluids Eng. 130, 034501 (2008).

${ }^{35}$ J. M. Smith, H. C. Van Ness, and M. M. Abbott, Introduction to Chemical Engineering Thermodynamics (McGraw-Hill, Singapore, 2001).

${ }^{36}$ H. P. Bloch and C. Soares, Turboexpanders and Process Applications (Gulf Professional Publishing, Boston, 2001) 
${ }^{37}$ T. Arts, M. Lambert de Rouvroit, and A. W. Rutherford, "Aero-thermal investigation of a highly loaded transonic linear turbine guide vane cascade," Von Karman Institute for Fluid Dynamics Technical Note No. 174, 1990.

${ }^{38}$ R. Span and W. Wagner, "A new equation of state for carbon dioxide covering the fluid region from the triple-point temperature to $1100 \mathrm{~K}$ at pressures up to 800 MPa," J. Phys. Chem. Ref. Data 25, 1509 (1996).

${ }^{39}$ R. Span and W. Wagner, "Equations of state for technical applications. II. Results for nonpolar fluids," Int. J. Thermophys. 24, 41 (2003).

${ }^{40} \mathrm{P}$. Colonna and S. Rebay, "Numerical simulation of dense gas flows on unstructured grids with an implicit high resolution upwind Euler solver," Int. J. Numer. Methods Fluids 46, 735 (2004). 